\title{
A PAPR REDUCTION TECHNIQUE IN OFDM SYSTEMS WITH A LARGE NUMBER OF SUBCARRIERS
}

\author{
Yasuhiro Shimazu $^{1}$, Yushi Shirato ${ }^{2}$ and Masahiro Muraguchi ${ }^{3}$ \\ ${ }^{1,3}$ Department of Electrical Engineering, Tokyo University of Science \\ 6-3-1 Niijuku, Katsushika-ku, Tokyo, 125-0051, Japan \\ ${ }^{2}$ NTT, Japan
}

\begin{abstract}
A major drawback of orthogonal frequency division multiplexing (OFDM) signals is extremely high peak-to-average power ratio (PAPR). Signals with high PAPR lead to a lowering of the energy efficiency of power amplifiers and the shortened operation time causes a serious problem in battery-powered wireless terminals. We have found the CAZAC precoding makes the $P A P R$ of M-array quadrature amplitude modulation (M-QAM) OFDM signals into the PAPR of $M-Q A M$ single-carrier signals. Therefore, it can dramatically improve the PAPR of OFDM signals. However, to satisfy the 3GPP-LTE specification of frequency spectrum, severe bandpass filtering of CAZAC-OFDM signal lead to unacceptable regrowth of the PAPR. The paper provides available control procedure for PAPR and spectrum managements. It is confirmed that the CAZAC-OFDM signal controlled by our procedure maintains enough low $P A P R$ and can provide comparable spectral specifications in the downlink channel of $3 G P P$ LTE standard.
\end{abstract}

\section{KEYWORDS}

OFDM, CAZAC, PAPR, spectral specifications

\section{INTRODUCTION}

Orthogonal frequency division multiplex (OFDM) systems that attain high speeds and high capacity have recently been attracting attention in wireless applications, e.g., wireless local area networks (WLANs), third generation partnership project long-term evolution (3GPP LTE), and the digital video broadcasting-terrestrial (DVB-T) standard [1], [2]. The main drawback of OFDM signal is its high peak-to-average power ratio (PAPR), which decreases the efficiency of power amplifiers (PAs) and increases transmitter power consumption [3]. Therefore, many techniques have been proposed to reduce the PAPR [4]. Well-known techniques are clipping-andfiltering, partial transmit sequences (PTSs), and selected mapping (SLM). The clipping-andfiltering limits the peak amplitude of the transmission signal. Since the technique, however, cut a portion of signal amplitude with important information, as a result, it causes non-linear distortion and degrades the BER performance. The PTS partitions input data into disjoint sub-blocks. Moreover, each sub-block is weighted by a phase factor. This technique chooses the phase factor to minimize the PAPR of combined signals. The SLM generates multiple candidate data blocks. All data blocks represent the same information. Although the PTS and the SLM can be expected to create a certain reduction in PAPR, both techniques need side information in the receiver, which decreases spectral efficiency. The most practical solution to improving PAPR is to 
introduce a single carrier scheme, i.e., single carrier frequency division multiplexing access (SCFDMA). The 3GPP LTE system adopts SC-FDMA for uplink multiple access systems [5]. However, SC-FDMA is not the best solution from the viewpoint of high spectrum utilization.

As the other option of PAPR reduction techniques, the constant amplitude zero auto-correlation (CAZAC) precoding had been proposed [6]. Afterwards we had found out that the CAZAC precoding (CAZAC-OFDM) made the PAPR of M-array quadrature amplitude modulation (MQAM) OFDM signals into the PAPR of M-QAM single-carrier signals [7]. Moreover, we had demonstrated the theoretical verification of the CAZAC-OFDM [8].

To put the CAZAC-OFDM into practical use, we are facing new complex problems. Although the CAZAC precoding dramatically improves the PAPR of OFDM signals, in order to satisfy the 3GPP-LTE specification of frequency spectrum, severe bandpass filtering of CAZAC-OFDM signal lead to unacceptable regrowth of the PAPR.

The paper provides available control procedure for PAPR and spectrum managements for the CAZAC-OFDM system. One simplest approach of improving the PAPR is to clip the regrowth amplitude of the signal to a fixed level. Here, as the regrowth amplitude has not important information, the BER performance does not been degraded. An accurate clipping boundary is easily defined, because we can manage the amplitude of the time-domain signal of CAZACOFDM like a single-carrier signal. As the correct demodulation of CAZAC-OFDM signals requires only values of the original signal points, we can improve the PAPR without any degradation of the BER and little degradation of the spectrum by the clipping. The CAZACOFDM scheme provides high-efficiency operation of power amplifiers, i.e., the Power-AddedEfficiency (PAE) of 38\%, the power consumption of the PAs becomes about one-third of those of conventional OFDM scheme, and it is comparable value of single carrier signal scheme, i.e., the SC-FDM scheme. Furthermore, although the PAPR of conventional OFDM scheme strongly deteriorates as increasing the number of subcarriers, the PAPR of the CAZAC-OFDM scheme does not depend on the number of subcarriers. We already confirmed the usefulness of CAZACOFDM for the case of 64 subcarriers, and in this paper, we examined CAZAC-OFDM using 1024 subcarriers closer to the LTE specification [9]. Therefore, we believe the CAZAC-OFDM scheme can use for uplink channels of future wireless mobile communication systems.

\section{CAZAC-OFDM SYSTEM}

\subsection{OFDM System}

In OFDM system, the frequency domain symbol $\boldsymbol{X}=\left[X_{0}, X_{1}, \ldots, X_{N-1}\right]^{T}$ is modulated by $N$ size inverse Fast Fourier Transform (IFFT). The discrete-time OFDM signal with $N$ subcarriers are represented as

$$
x_{n}=\sum_{k=0}^{N-1} X_{k} e^{j 2 \pi k n / N}
$$

where $j=\sqrt{-1}$ and $n$ is discrete time index. On the other hand, receiver acquires frequency domain symbol $\boldsymbol{Y}$ by applying FFT to received signal $\boldsymbol{y}$.

$$
Y_{k}=\sum_{n=0}^{N-1} y_{n} e^{-j 2 \pi k n / N}
$$




$$
=\sum_{n=0}^{N-1}\left(x_{n}+\text { Noise }\right) e^{-j 2 \pi k n / N} \text {. }
$$

The PAPR of the OFDM signal eq. (1) can be expressed as

$$
P A P R=\frac{\max _{0 \leq n \leq N-1}\left|x_{n}\right|^{2}}{\mathrm{E}\left[\left|x_{n}\right|^{2}\right]},
$$

where E[·] is expectation operator. PAPR represents amplitude fluctuation of each symbol. To improve the accuracy of PAPR, the OFDM signal $x_{k}$ is converted to $L$-times oversampled time domain signal.

As shown from eq. (2), the OFDM signal is composed of a plurality of subcarrier signals, which causes an increase in amplitude fluctuation. A high PAPR signal increases the Input Back Off (IBO) at the power amplifier to amplify the transmit signal without distortion. In general, increasing in IBO causes decreasing the efficiency of PA [3].

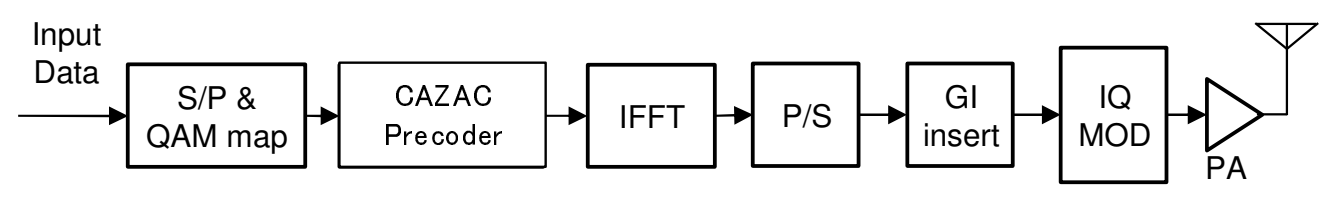

(a) Transmitter

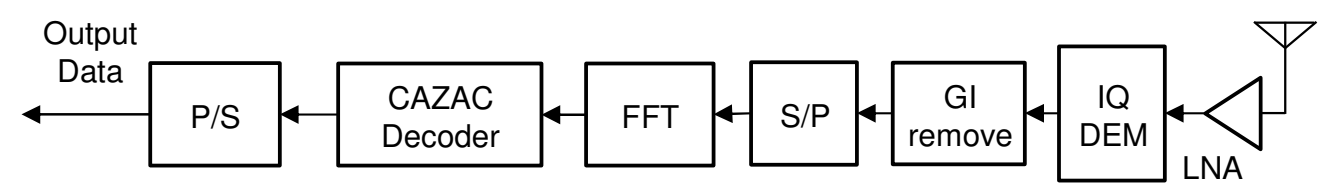

(b) Receiver

Figure 1.CAZAC-OFDM system

\subsection{CAZAC Precoding Technique}

Figure 1 shows a transmitter and receiver block diagrams for a CAZAC-OFDM system. The CAZAC sequence is an orthogonal sequence and the autocorrelation function is a delta function. In addition, the CAZAC sequence has a characteristic that the amplitude exhibits a constant value in both time and frequency domain. Zadoff-Chu (ZC) sequences is one of the CAZAC sequence. The ZC sequence $C_{k}$ of length $L$ is defined as

$$
C_{k}=\left\{\begin{array}{l}
\exp \left(\frac{j \pi k(k-1) r}{L}\right)(L \text { is even }) \\
\exp \left(\frac{j \pi(k-1)^{2} r}{L}\right)(L \text { is odd })
\end{array}\right.
$$


Where $L$ is the length of the CAZAC sequence and $r$ is the sequence number, $k=1,2, \cdots L . L$ is a natural number, and $r$ is a prime integer with respect to $L$. CAZAC sequences are generated by cyclic shift of the original CAZAC sequence. The periodic cross-correlation function $\rho$ is defined as

$$
\begin{aligned}
\rho(m) & =\sum_{n=1}^{L-1} c_{n} c_{(c-m) \bmod L}^{*} \\
& =\left\{\begin{array}{l}
L(m=0) \\
0(m \neq 0)
\end{array}\right.
\end{aligned}
$$

Where $m$ is integer variables.

CAZAC precoding uses a square matrix $M$ generated from the equation in the case where $\mathrm{L}$ in equation (4) is an even number. The matrix equation is defined as

$$
M=\frac{1}{\sqrt{N}}\left[\begin{array}{cccc}
c_{1} & c_{2} & \cdots & c_{N} \\
c_{N+1} & c_{N+2} & \cdots & c_{2 N} \\
\vdots & \vdots & \ddots & \vdots \\
c_{(N-1) N+1} & \cdots & \cdots & c_{N^{2}}
\end{array}\right]
$$

Where matrix $M$ is the rearrangement of in equation (4) in the row direction, and $N$ is the number of subcarriers and $L=N^{2}, r=1 . N$ is an even number, so $L$ is also an even number.

Multiply the signal before IFFT by the matrix calculate the product of the transposed the frequency domain symbol $X=\left[X_{1}, X_{2}, \cdots, X_{N}\right]$ and the matrix $M$, and create a CAZAC equalized signal $X^{\prime} . X$ 'is represented as

$$
\begin{gathered}
X^{\prime}=M \cdot X^{T} \\
=\frac{1}{\sqrt{N}}\left[\begin{array}{ccc}
c_{1} & \cdots & c_{N} \\
c_{N+1} & \cdots & c_{2 N} \\
\vdots & \ddots & \vdots \\
c_{(N-1) N+1} & \ldots & c_{N^{2}}
\end{array}\right] \cdot\left[\begin{array}{c}
X_{1} \\
X_{2} \\
\vdots \\
X_{N}
\end{array}\right]
\end{gathered}
$$

An IFFT operation is performed on this signal. The OFDM time domain signal $x_{n}$ of the sample is represented as

$$
\begin{aligned}
x_{n}= & \frac{1}{N} \sum_{k=0}^{N-1} X_{k}^{\prime} e^{j 2 \pi k n / N} \\
& =\sum_{k=0}^{N-1}\left\{\sum_{m=0}^{N-1} e^{j \pi(m+k N)^{2} / L} X_{m}\right\} e^{j 2 \pi k n / N} \\
= & \sum_{m=0}^{N-1} e^{j \pi m^{2} / N^{2}} X_{m}\left\{\sum_{k=0}^{N-1} e^{j 2 \pi k(m+n) / N} e^{j \pi k^{2}}\right\}
\end{aligned}
$$

Where $k$ is an integer not less than 0 , so the following equation is developed. 


$$
\exp \left(j \pi k^{2}\right)=\left\{\begin{array}{cc}
1 & (k: \text { even }) \\
-1 & (k: \text { odd })
\end{array}\right.
$$

To lead the equation (10) from the equation (9).

$$
\exp \left(j \pi k^{2}\right)=(-1)^{k}
$$

Substituting the equation (10) into the equation (8) lead to the equation (11).

$$
x_{n}=\sum_{m=0}^{N-1} e^{j \pi m^{2} / N^{2}} X_{m}\left\{\sum_{k=0}^{N-1}\left\{-e^{j 2 \pi(m+n) / N}\right\}^{k}\right\}
$$

The inside of \{\} in equation (11) is the sum of the geometric progression. Therefore, equation (12) is derived.

$$
\sum_{k=0}^{N-1}\left\{-e^{j 2 \pi(m+n) / N}\right\}^{k}=\left\{\begin{array}{l}
N\left(-e^{j 2 \pi(m+n) / N}=1\right) \\
0\left(-e^{j 2 \pi(m+n) / N} \neq 1\right)
\end{array}\right.
$$

When $2 \pi(m+n) / N=1,2(m+n) / N$ is an integer and odd number. Also, $n$ and $m$ are $0 \leq n \leq$ $N-1,0 \leq m \leq N-1$. These relationships are satisfying the relationship of equation (13).

$$
m=\frac{\mathrm{N}}{2}-n(\bmod N)
$$

Equation (14) is derived from equations (11), (12) and (13).

$$
\begin{gathered}
x_{n}=e^{j \pi\{\mathrm{N} / 2-n(\bmod N)\}^{2} / N^{2}} X_{\frac{N}{2}-n(\bmod N)} \\
=\mathrm{c}_{\frac{N}{2}-n(\bmod N)} X_{\frac{N}{2}-n(\bmod N)}
\end{gathered}
$$

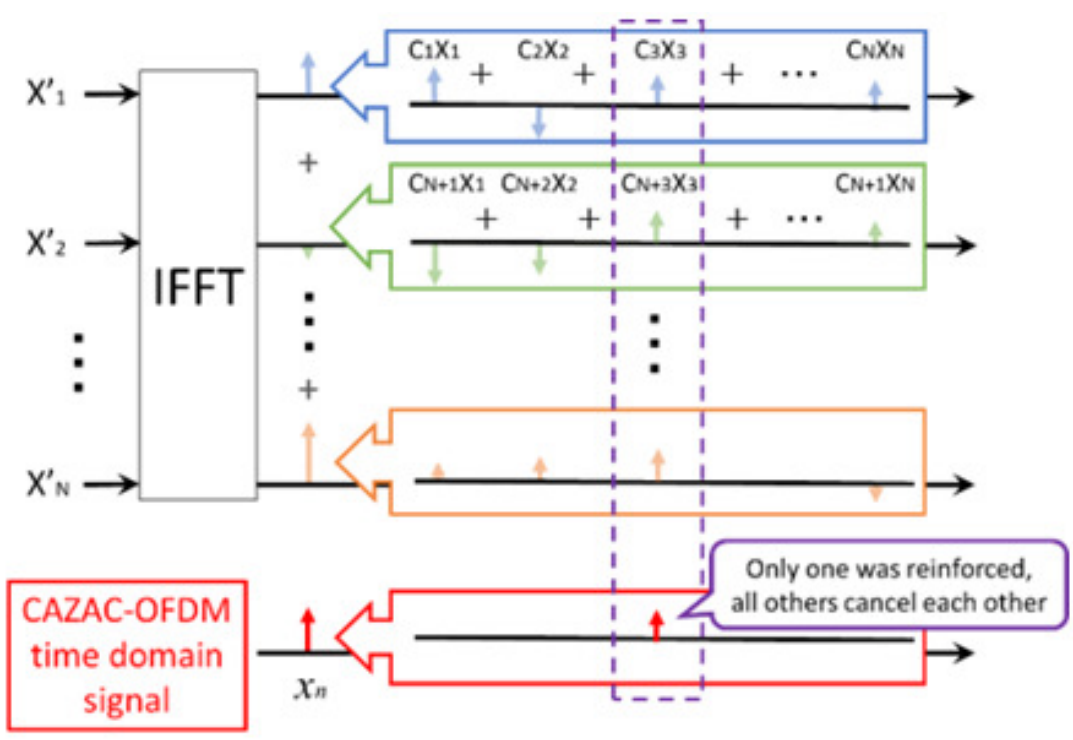

Figure 2.The time domain signal of CAZAC-OFDM ${ }^{[8]}$ 


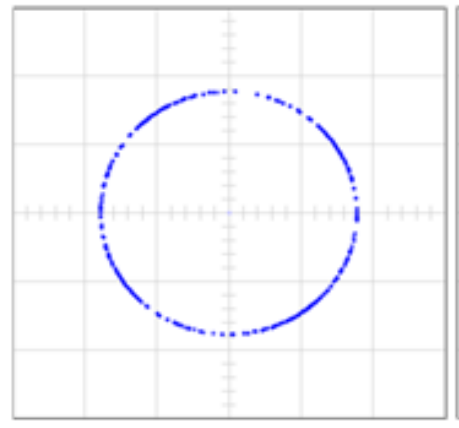

(a) QPSK

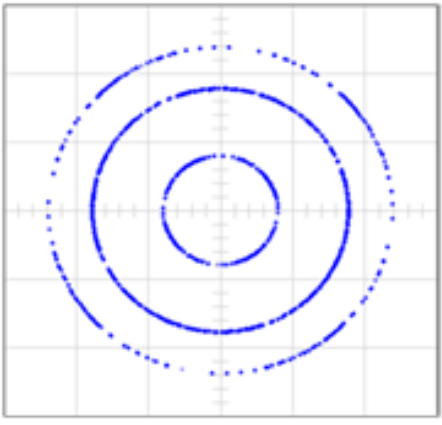

(b) 16QAM

Figure 3.The time domain signal points of the CAZAC-OFDM Signals on the complex phase plane.

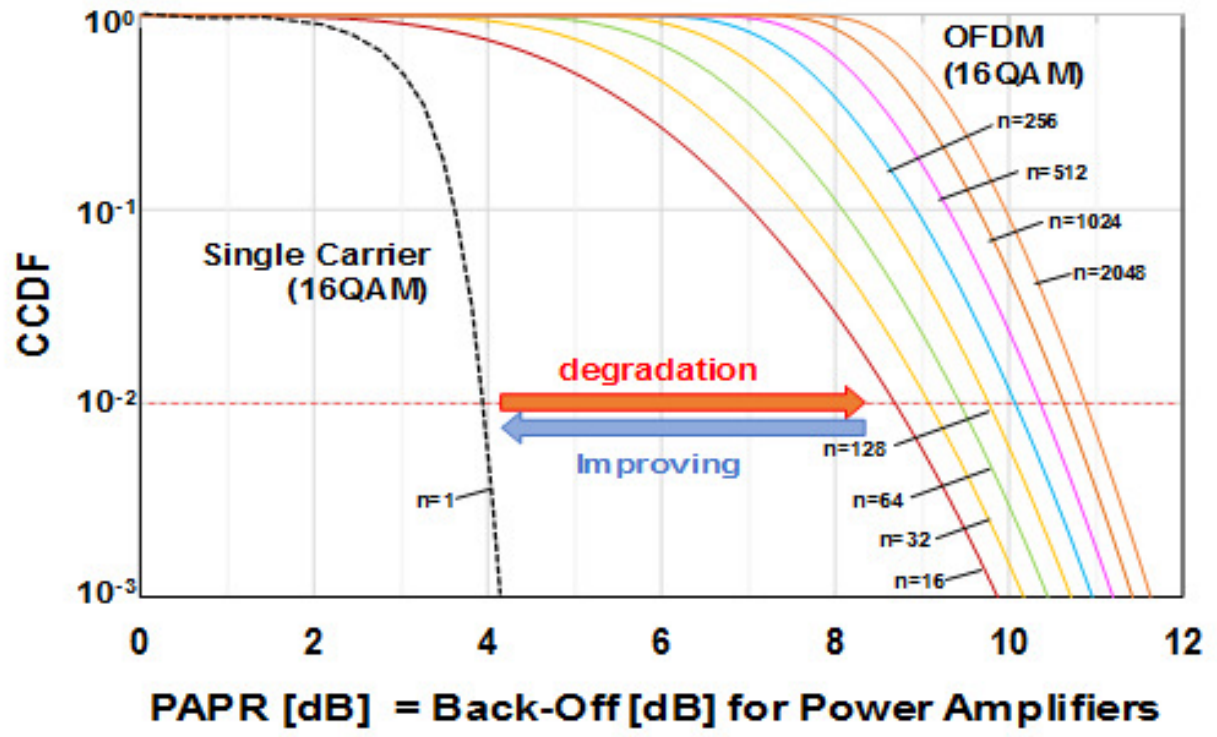

Figure 4. Demerit of increasing number of subcarriers in OFDM

Equation (14) shows that CAZAC precoding converts the PAPR of the OFDM time domain signal into the PAPR of a single-carrier signal. Figure 2 shows the image diagram of the CAZACOFDM time domain signal. Among the components of the coefficient $X$, only one was reinforced, all others cancel each other.

Figure 3 (a) and (b) show the time domain signal points of CAZAC-OFDM, which shows the IQMOD input in Fig. 1. The discrete-time signal points of QPSK CAZAC-OFDM line up on the unit circle orbit as shown in Fig. 3 (a), and the discrete-time signal points of 16QAM CAZACOFDM signal are on the three concentric circle orbits as shown in Fig. 3 (b).

Figure 4 shows demerit of increasing number of subcarriers in OFDM. In the conventional OFDM, PAPR also deteriorates as the number of subcarriers increases, but CAZAC-OFDM can improve PAPR as much as single carrier without depending on the number of subcarriers. This characteristic is very suitable for the recent trend of increasing the communication speed by increasing the number of subcarriers. 


\section{SPECTRAL CONTROL}

\subsection{Procedure of Spectral Control}

To make the discrete-time OFDM signal with the proper pass band characteristics, we set the subcarrier allocation as shown in Fig.5. This process is equivalent to severe filtering. That is, while spectral control can be performed, transient response occurs between signal points. However, due to oversampling, this transient response part appears as an interpolation point between the original signal points in the time domain. Here, we set the FFT size of CAZACOFDM to 1024. Therefore, four times interpolated (oversampled) FFT size becomes to 4096 and three interpolation points are inserted between the original signal points.

The CAZAC-OFDM signal at the IQ-MOD output in Fig. 1 is an analog signal wave which deals with the pass band signal with a carrier frequency of $f_{\mathrm{c}}$ in the continuous time domain. Since $f_{\mathrm{c}}$ in general is much higher than $1 / T_{s}$, a continuous-time base band OFDM signal with the symbol period $T_{\mathrm{s}}$ and the corresponding pass band signal with the carrier frequency $f_{\mathrm{c}}$ have almost the same waveform. However, in general, the waveform for the discrete-time baseband signal may not be the same as that for the continuous-time baseband signal. In practice, the PAPR for the continuous-time baseband signal can be measured only after implementing the actual hardware, including digital-to-analog convertor (DAC).

In other words, measurement of the PAPR for the continuous-time baseband signal is not straightforward. Therefore, there must be some means of estimating the PAPR from the discretetime signal. Fortunately, it is known that the discrete-time baseband signal can show almost the same PAPR as the continuous-time baseband signal if it is four times interpolated (oversampled) [9].

The first task to consider is that the CAZAC-OFDM spectrum of 1024 subcarriers with the bandwidth $(\mathrm{BW})$ of $18 \mathrm{MHz}$ is centered on the carrier frequency of $f_{\mathrm{c}}, 27 \mathrm{MHz}$ bandwidth to the left, i.e., the space of 1536 null subcarriers, and in the same way $27 \mathrm{MHz}$ bandwidth to the right.

A block diagram of the generation of CAZAC-OFDM symbols is shown inFig.6.

The second step to produce the signal is to apply a transmit filtering with proper roll-off by a FFT-window processing, and to avoid aliasing by low-pass filtering.

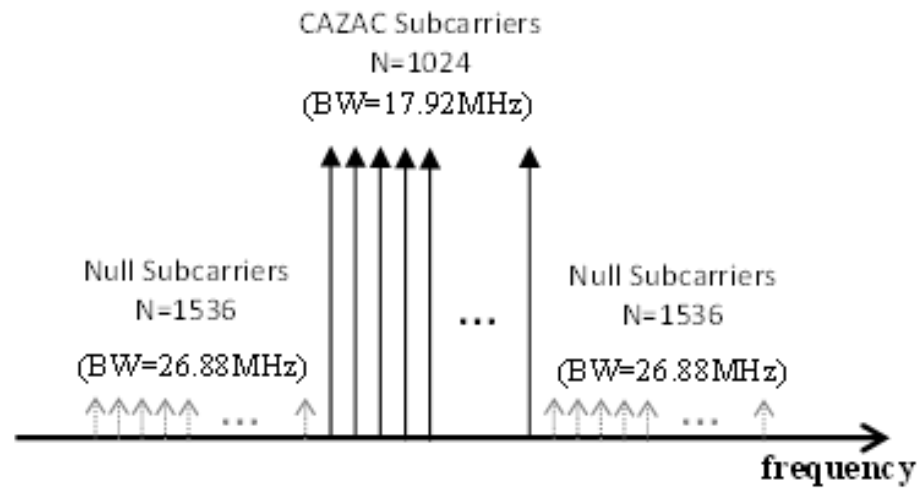

Figure 5. Subcarrier allocation based on four times interpolated (oversampled) FFT processing. 
Table I. Major Simulation Parameters

\begin{tabular}{|c|c|}
\hline Number of CAZAC Subcarriers & 1024 [Data: 1019, Pilot: 5] \\
\hline IFFT \& FFT size & 4096 \\
\hline Symbol Period $T_{s}$ & $57 \mu \mathrm{sec}$ \\
\hline Guard Interval & $5 \mu \mathrm{sec}$ \\
\hline Modulation of Subcarriers & 16QAM \\
\hline Data Rate & $65.74 \mathrm{Mbps}$ \\
\hline Channel Model & $\begin{array}{l}\text { AWGN } \\
\text { Flat Rayleigh Fading } \\
\text {-Doppler Frequency }\left(f_{D} T_{S}\right): 8.6 \times 10^{-4} \\
\text { Frequency Selective Fading } \\
\text {-Notch Filter } \\
\quad \text { Center frequency } 0 \mathrm{~Hz} \\
\quad \text { Bandwidth } 70 \mathrm{kHz} \\
\text { Gain }-7 \mathrm{dBm}\end{array}$ \\
\hline
\end{tabular}

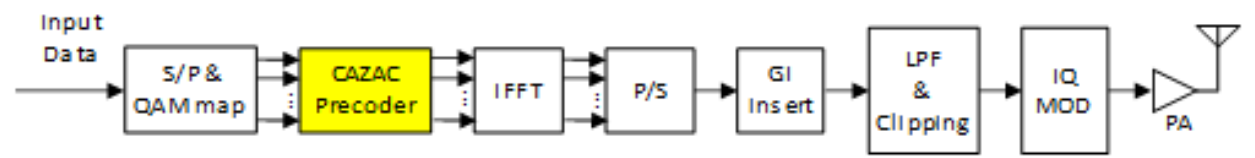

Figure 6. The CAZAC-OFDM transmitter based on four times interpolated (oversampled) FFT processing.

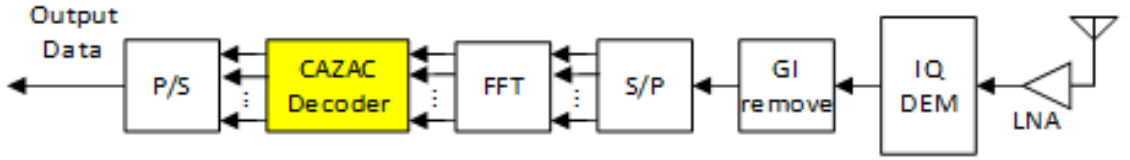

Figure 7. The receiver block diagram for spectral controlled CAZAC-OFDM signal.

\subsection{Simulation Results}

We carried out our simulation with the MATLAB/Simulink to evaluate the performance of the system. Table I summarizes the simulation parameters.

Figure 8 shows the spectrum for the 16QAM CAZAC-OFDM system when oversampling and filtering are performed. It is confirmed that the spectral performance satisfies the 3GPP LTE specifications. 


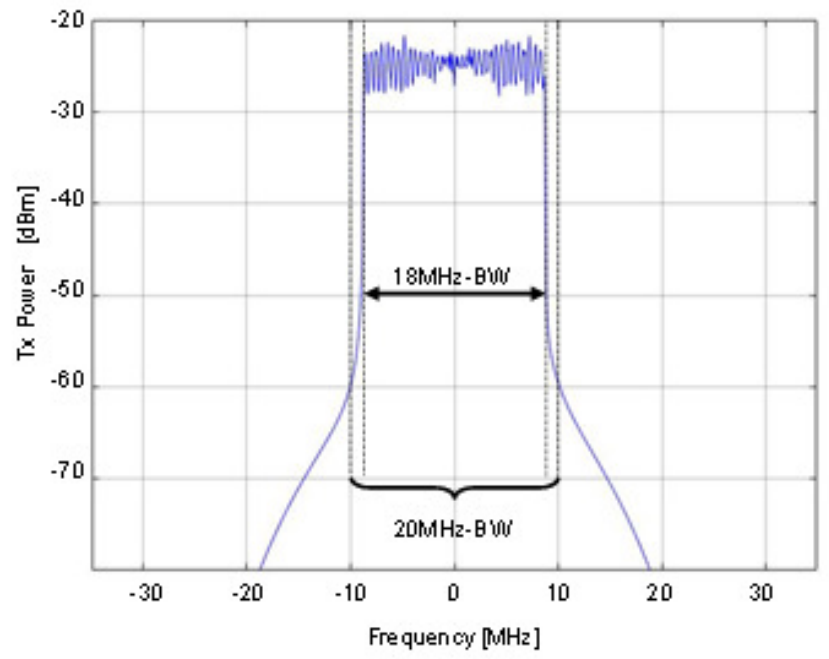

Figure 8 . The spectrum controlled 16QAM CAZAC-OFDM signal.

\section{PAPR CONTROL}

\subsection{Procedure of PAPR Control}

The CAZAC precoding scheme makes the PAPR of M-array quadrature amplitude modulation (M-QAM) OFDM signals into the PAPR of M-QAM single-carrier signals [7]. Therefore, it dramatically improves the PAPR of OFDM signals. However, an aggressive spectral control results in a high level of correction required in the out of band region, resulting in greater peak regrowth and lead to unacceptable degradation of the PAPR [9].

Figure 9 shows the amplitude of band pass and four times oversampled QPSK CAZAC-OFDM signal with subcarrier allocation shown in Fig. 5. Here, it is known that actual analog signal, i.e., continuous-time signal, can be simulated by discrete-time signal with four times oversampling. The original sample points of QPSK are all the same amplitude. However, the interpolation point generated after spectral shaping causes amplitude fluctuation. This means obvious degradation of the PAPR.

One simplest approach of improving the PAPR is to clip amplitude of interpolating points over to a fixed level, because the amplitude of interpolating points has not important information. Correct demodulation of CAZAC-OFDM signals requires only values of the original sample points, and it is possible to skip out the values of the interpolating points. Therefore, if the clipping boundary is greater than or equal to the maximum value of the original sample point, it can be clipped without affecting the BER performance. 


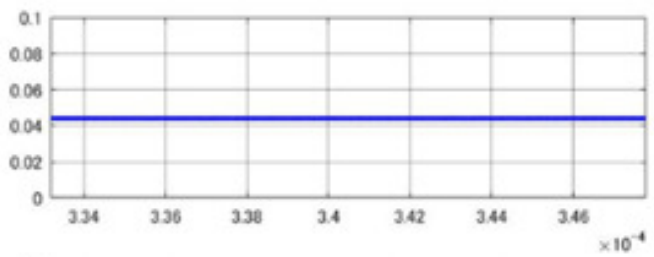

Before

Spectral Control

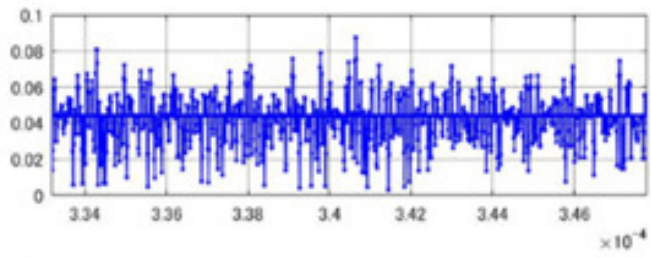

After

Spectral Control

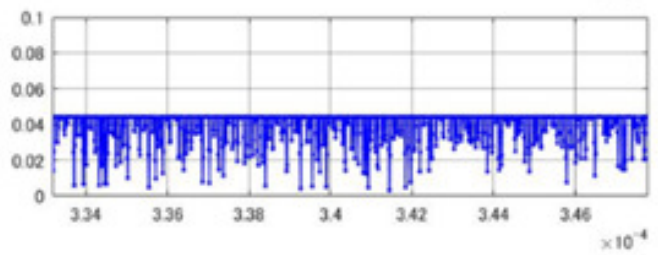

\section{After}

Clipping

Figure 9. Amplitude of band pass and oversampled QPSK CAZAC-OFDM signal in the time domain.

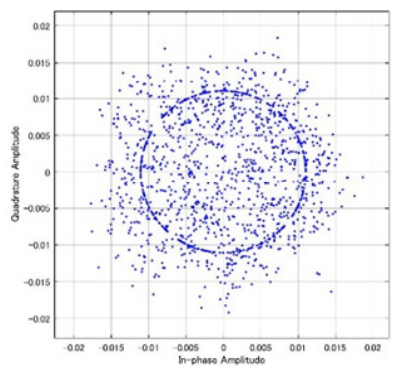

(a) QPSK

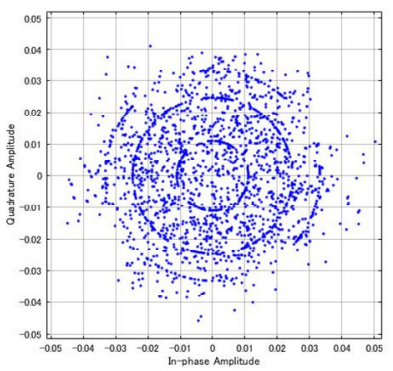

(b) 16QAM

Figure 10.The time domain signal points of bandpass and oversampled CAZAC-OFDM signal on the complex phase plane.

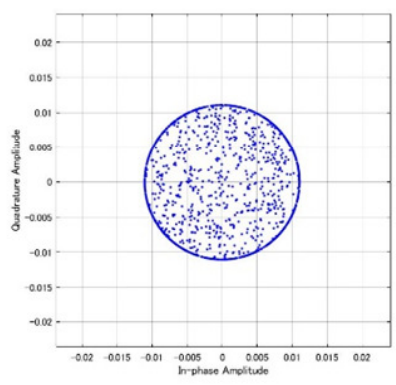

(a) QPSK

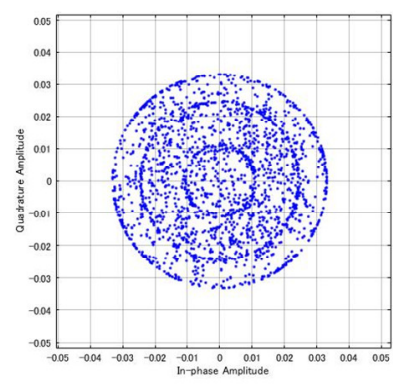

(b) 16QAM

Figure 11.The time domain signal points of band pass and oversampled CAZAC-OFDM signal with clipped amplitude processing. 


\subsection{Simulation Results}

Figure 10 shows the time domain signal points of band pass and four times over sampled CAZAC-OFDM signal with subcarrier allocation shown in Fig. 5. The original sample points of 16QAM CAZAC-OFDM signal are on the three concentric circle orbits as shown in Fig. 3 (b). However, many of the interpolating points overfly to outer side of the largest circle orbit. This means obvious degradation of the PAPR.

As noted previously, correct demodulation of CAZAC-OFDM signals requires only values of the original sample points, which are all in the inner-side of the largest circle orbit of 16QAM CAZAC-OFDM signal, and it is possible to clip amplitude of the interpolating sample points. Figure 11 shows the time domain signal points of band pass and oversampled 16-QAM CAZACOFDM signal with clipped amplitude processing.

We first considered the complementary cumulative distribution function (CCDF) of PAPR to evaluate the performance of PAPR, which is the probability that PAPR will be higher than a certain PAPR value PAPR0, i.e., Pr (PAPR > PAPR0). Figure 12 plots the CCDF of PAPR of 16QAM CAZAC-OFDM system with and without clipping, as well as the conventional 16QAM OFDM and single-carrier 16QAM systems. We found that the PAPR of the 16QAM CAZACOFDM system with clipping was almost equal to that of the single-carrier 16QAM system. Moreover, the PAPR of the 16QAM CAZAC-OFDM system with clipping was improved by 6.3 $\mathrm{dB}$ at the CCDF of $10^{-2}$ compared with the conventional 16QAM OFDM system.

Figure 13 shows spectrum for the 16QAM CAZAC-OFDM system with clipping. The spectral performance looks acceptable although a little degradation is observed. The result indicates the clipping does not bring serious effect to the spectral performance.

We next examined the bit error rate (BER) of the proposed system. We considered the additive white Gaussian noise (AWGN). Figure 14 shows the BER performances of the 16QAM CAZACOFDM systems with clipping which are applied for AWGN channels. The results indicate that the CAZAC-OFDM system with clipping does not degrade BER performances.

Then, flat Rayleigh fading was considered as a fading channel and a notch filter was considered as a frequency selective channel. This BER characteristic is shown in Figure 15. For the flat fading channel, CAZAC-OFDM was improved by about $6 \mathrm{~dB}$ at the BER of $10^{-3}$ compared with the conventional OFDM. Also, in the frequency selective channel, a certain error occurs in the conventional OFDM even in a portion with a high SNR. However, in CAZAC-OFDM, since the information of each subcarrier is dispersed throughout the signal by the CAZAC precoder, it has a frequency diversity effect, and even if a specific frequency is lost, the influence becomes small.

Figure 16 shows PAPR vs. Power Added Efficiency in typical PA in the frequency band of $800 \mathrm{MHz}-2 \mathrm{GHz}$ [10]. For a linear amplification, PAs should be operated at the point back off from the saturation point, i.e., the $1 \mathrm{~dB}$-gain-suppression-pint to be exact. Here, the value of the back off should correspond to the PAPR of the input signal for a linear amplification. As a large value of the back off brings degradation of PAE, the PAE in the conventional OFDM scheme becomes only $14 \%$. On the other hand, it is expected that the PAE in the CAZAC-OFDM scheme with clipping is $38 \%$. This is comparable value of single carrier signal scheme, i.e., the SC-FDM scheme. 


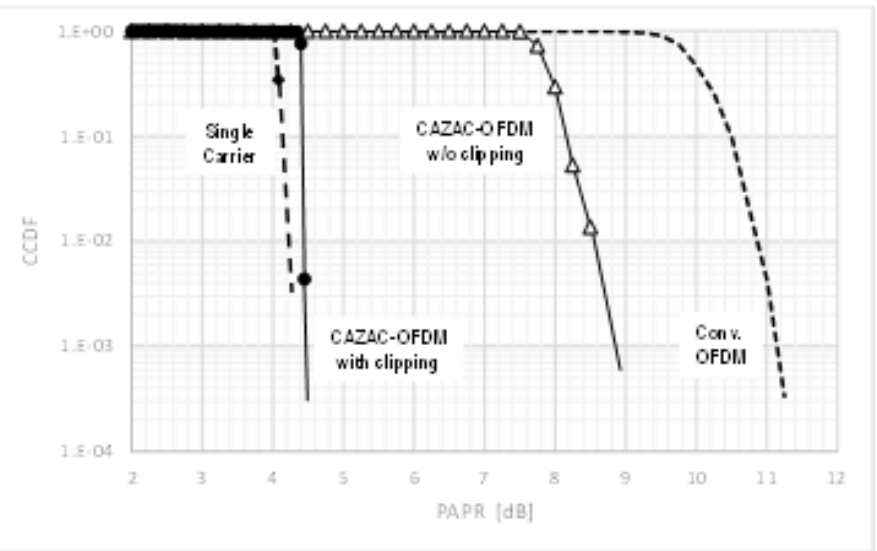

Fig. 12. PAPR of the proposed CAZAC-OFDM.

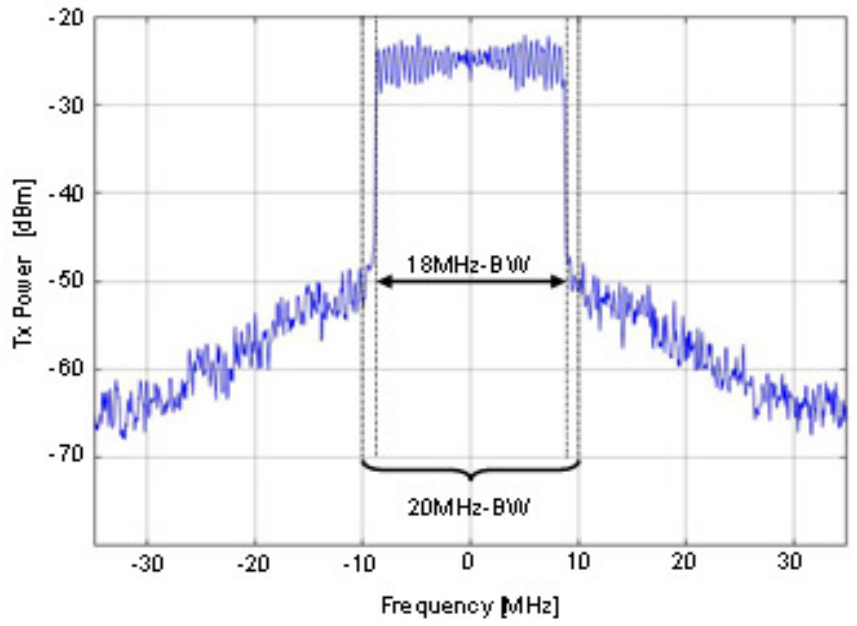

Fig. 13. Spectrum of the 16QAM CAZAC-OFDM Signal with clipping.

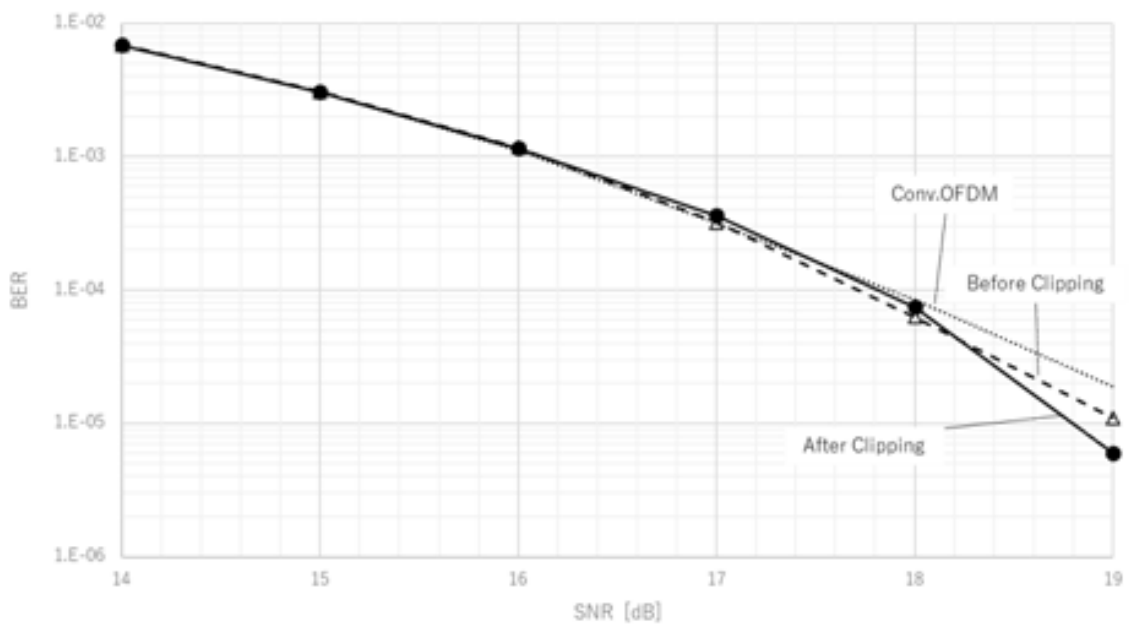

Fig. 14. BER performances of the CAZAC-OFDM under AWGN channel. 


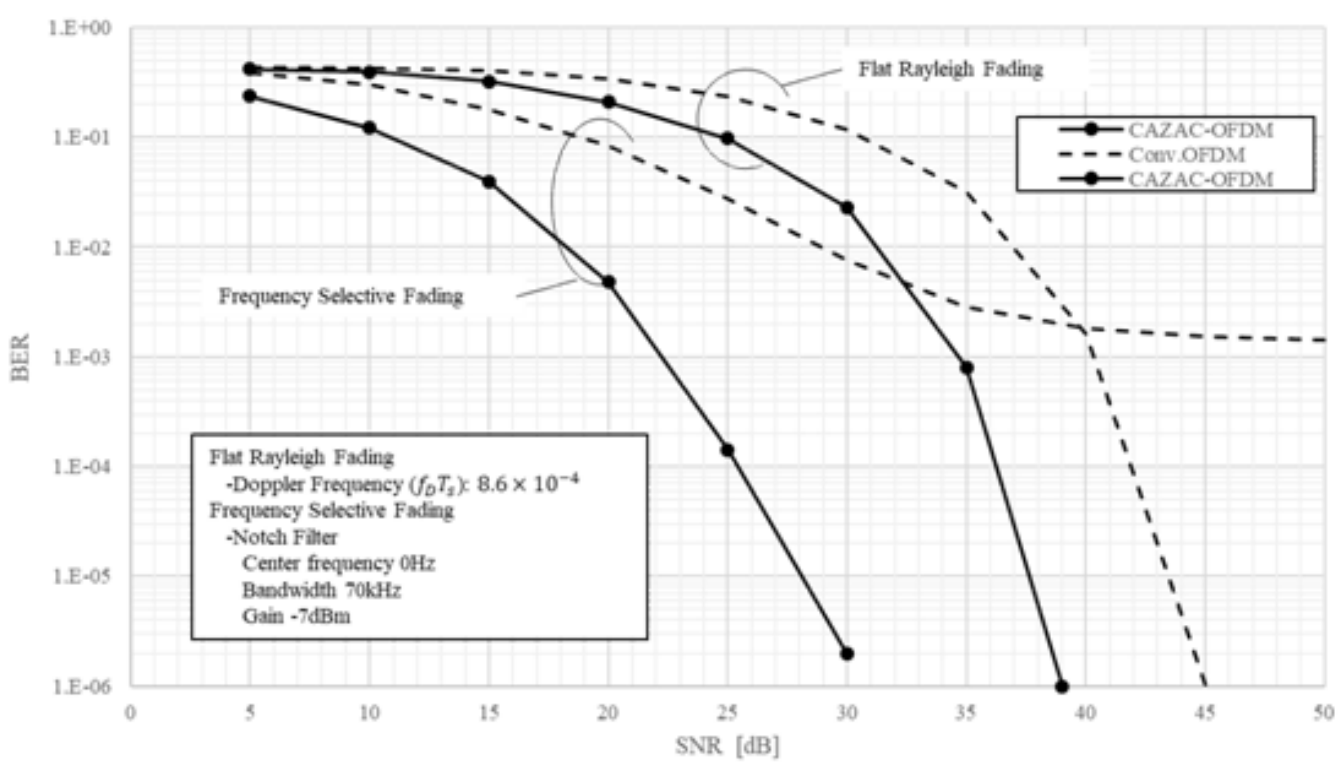

Fig. 15. BER performances of the CAZAC-OFDM under Fading Channel

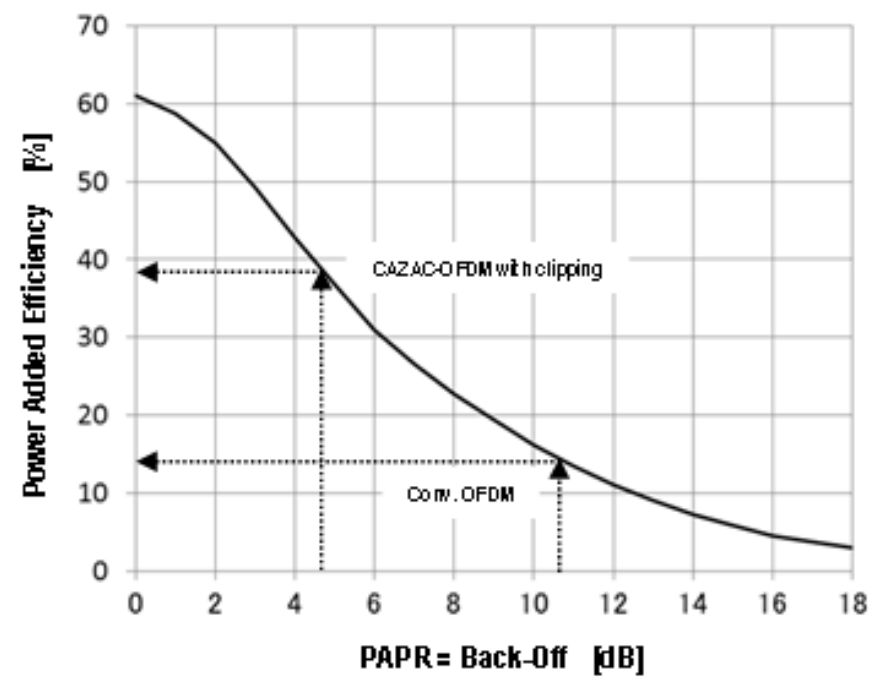

Fig. 16. PAPR vs. Power Added Efficiency of the PA ${ }^{[10]}$. 
Table II. Comparisons of PAPR, PAE and Power Consumption of the $\mathrm{PA}^{[10]}$ with each waveform.

\begin{tabular}{|l|l|l|l||l|}
\hline $\begin{array}{l}\text { Waveform } \\
(16 \mathrm{Q} A M)\end{array}$ & $\begin{array}{c}\text { PAPR [dB] } \\
\text { @ CCDF=1\% }\end{array}$ & $\begin{array}{l}\text { PAPR Reduction } \\
{[\mathrm{dB}]}\end{array}$ & $\begin{array}{c}\text { PAE } \\
{[\%]}\end{array}$ & $\begin{array}{l}\text { Power Consumption } \\
\text { @PTx:200mW [mW] }\end{array}$ \\
\hline $\begin{array}{l}\text { CAZAC-OFDM } \\
\text { with clipping }\end{array}$ & 4.5 & 6.3 & 38 & 526 \\
\hline $\begin{array}{l}\text { CAZAC-OFDM } \\
\text { w/o clipping }\end{array}$ & 8.5 & 2.3 & 20 & 1000 \\
\hline Single Carrier & 4.2 & 6.6 & 40 & 500 \\
\hline Conv. OFDM & 10.8 & - & 14 & 1429 \\
\hline
\end{tabular}

\section{CONCLUSIONS}

The paper provides available control procedure for PAPR and spectrum managements for the CAZAC-OFDM system. One simplest approach of improving the PAPR is to introduce the fourtimes oversampling technique and to clip the amplitude of the interpolating points to a fixed level. An accurate clipping boundary is easily defined because we can manage the amplitude of the time-domain signal of CAZAC-OFDM like a single-carrier signal. As the correct demodulation of CAZAC-OFDM signals requires only values of the original signal points, we can improve the PAPR without any degradation of the BER and a little degradation of the spectrum by the clipping. The CAZAC-OFDM scheme provides high-efficiency operation of power amplifiers, i.e., the PAE of $38 \%$, the power consumption of the PAs becomes about one-third of those of conventional OFDM scheme, and it is comparable value of single carrier signal scheme, i.e., the SC-FDM scheme. Furthermore, although the PAPR of conventional OFDM scheme strongly deteriorates as increasing the number of subcarriers, the PAPR of the CAZAC-OFDM scheme does not depend on the number of subcarriers.

Therefore, we believe the CAZAC-OFDM scheme can use for uplink channels of future wireless mobile communication systems.

\section{REFERENCES}

[1] IEEE, "Part 11: Wireless LAN Medium Access Control (MAC) and Physical Layer (PHY) Specifications,” IEEE Std 802.11-2012 (Revision of IEEE Std 802.11-2007), pp. 1-2695 Mar. 2012.

[2] A. Ghosh, R. Ratasuk, B. Mondal, N. Mangalvedhe, and T. Thomas, “ LTE-advanced: Nextgeneration Wireless Broadband Technology," IEEE Wireless Communications, vol. 17, no. 3, pp. 1022, June 2010.

[3] J. Joung, C. K. Ho, K. Adachi, and S. Sun, "A Survey on Power-Amplifier-Centric Techniques for Spectrum- and Energy-Efficient Wireless Communications," IEEE Communications Surveys \& Tutorials, vol. 17, no. 1, pp. 315-333, Jan. 2015.

[4] H. Seung, Hee and L. Jae, Hong, "An Overview of Peak-to-average Power Ratio Reduction Techniques for Multicarrier Transmission," IEEE Wireless Communications, vol. 12, no. 2, pp. 5665, Apr. 2005.

[5] H. Myung, J. Lim, and D. Goodman, "Single Carrier FDMA for Uplink Wireless Transmission," IEEE Vehicular Technology Magazine, vol. 1,no. 3, pp. 30-38, Sep. 2006. 
[6] I. Baig and V. Jeoti, "PAPR Reduction in OFDM Systems: Zad off-Chu Matrix Transform Based Pre/Post-Coding Techniques," in Proc.of the 2nd International Conference on Computational Intelligence, Communication Systems and Networks, pp. 373-377, July 2010.

[7] R. Ishioka, T. Kimura and M. Muraguchi, "A Proposal for a New OFDM Wireless System using a CAZAC Precoding Scheme,” Proc. AICT 2017, pp. 47-51, June 2017.

[8] Kazuki Miyazawa, Tomotaka Kimura, Masahiro Muraguchi, "Proposal of visible light OFDM system with CAZAC equalization,” 23rd Asia-Pacific Conference on Communications (APCC), pp.491-496, Dec. 2017.

[9] Yoshitsugu Sugai, Yushi Shirato, Tomotaka Kimura and Masahiro Muraguchi, "PAPR and Spectral Control Procedure for OFDM Wireless Systems Using CAZAC Equalization," The Fourteenth Advanced International Conference on Telecommunications (AICT) 2018, pp.75-80, July 2018.

[10] H. Okazaki, A. Fukuda, and S. Narahashi, "Band-reconfigurable high-efficiency power amplifier: 900MHz/1900MHz dual-band PA using MEMS switches,” NTT DoCoMo Tech. J., vol. 7, no. 1, pp. 11-18, Jun. 2005. 